\title{
A Study to Assess the Efficacy of Magnetization Transfer Ratio in Differentiating Tuberculoma From Neurocysticercosis
}

\author{
Dr. Ganga Devi Rajapandian ${ }^{1}$, Dr. Shivashankar Kumaresan ${ }^{1}$, Dr. Babupeter ${ }^{2}$, \\ Dr. Priya Muthaiyan ${ }^{3}$ \\ ${ }^{I}$ Associate Professor, Formerly Assistant Professor, Stanley Medical College, Chennai, India, \\ ${ }^{2}$ Associate Professor Madras Medical College, Chennai, India, \\ ${ }^{3}$ Assistant Professor Stanley Medical College, Chennai, India.
}

\begin{abstract}
Background: In Indian patients with acute onset seizures, solitary ring enhancing lesion is the most common imaging abnormality in contrast enhanced CT brain. The two most common cause of these CT-detected lesions are neurocysticercosis (NCC) and tuberculoma. Magnetization transfer ratio obtained using magnetization transfer MR imaging can differentiate tuberculoma from NCC.

Methods: The study group included 43 consecutive patients with intra cranial ring enhancing lesions who were referred for MRI Brain. Conventional spin echo, non contrast T1-Weighted (TR 1000, TE 14) axial MR images with and without off - resonance saturation pulse and T2WI were taken for all patients. 3D CISS sequence was taken in axial plane at the site of the lesion. Signal intensity from the rim of the granuloma was obtained with a single pixel from the conventional T1WI (without an off resonance pulse) and MT images (with an off resonance pulse). For each region of interest (ROI), MTR is calculated from the two images using the formula,

$M T R=(M o-M s) \times 100$

Mo

where Mo, Ms represent the signal intensity with the saturation pulse off and on, respectively. Results : The MT Ratio obtained from the rim of the lesion was tabulated and analyzed using ANOVA method. The Mean MTR was obtained for each group. Mean MTR for tuberculoma was 16.6 +/- 3.1(15.6 -17.56). Innocuous cystic lesions (representing vesicular stage of NCC) showed mean MTR of $10.9 \pm 2.8$ (9.6 - 12.18). Degenerative NCC showed MTR of $20.8 \pm 3.5$ (18.7 - 22.9).There were 3 NCC which were hypointense on T2WI which showed a mean MTR which was significantly higher (23.72 \pm 3$)$.

Conclusions:MT ratio can be used to differentiate T2 hypointense NCC and Tuberculoma. MT Ratio of Tuberculoma is $16.6 \pm 3.1$. MT Ratio of T2 hypointense NCC is $23.725 \pm 3$. Including precontrast MT SE T1 Weighted sequence in our routine MRI Brain protocol for granuloma imaging can help us differentiate T2 hypointense NCC and Tuberculoma, better visualize T2 invisible Tuberculomas and detect presence of associated meningeal disease.
\end{abstract}

Keywords: Tuberculoma, Neurocysticercosis, Magnetization transfer MR imaging, Magnetization transfer ratio, 3D CISS, Scolex.

\section{Introduction}

In Indian patients with acute onset seizures, solitary ring enhancing lesion is the most common imaging abnormality in contrast enhanced CT brain (1 - 7). In 1980's ring enhancing lesions were presumed to be tuberculoma and treated with empirical Anti tubercular treatment. A major breakthrough came, when Chandy et al (1989), followed by Rajasekhar et al (1993) found on stereo tactic biopsy and histopathological studies that Neurocysticercosis is the commonest etiology of single enhancing lesion in CT scans $(3,9)$. The second most common cause of these CT-detected lesions is tuberculoma. Patients with both tuberculosis and NCC have similar clinical and neuroimaging features. Using CECT and conventional MRI Brain, tuberculoma and neurocysticercus granuloma can be differentiated to some extent(3).Various studies have shown that magnetization transfer ratio obtained using magnetization transfer MR imaging can differentiate tuberculoma from $\mathrm{NCC}(8)$.

Magnetization Transfer MR Imaging is a technique for improving image contrast in Magnetic Resonance (MR) imaging (11).It is based on application of off-resonance radio-frequency pulses and observing their effects on MR images. There are numerous applications of MT imaging (11, 12, 15, 22, 23, 24). The utilization of magnetization transfer was extended to clinical imaging, including its use with gradient-echo imaging and MR angiography $(15,20,21)$. It has also been used with short repetition time (TR) images to detect contrast enhancement and other regions containing short T1 elements, including methemoglobin (11) and lipid. It has been proposed as the reason that adult white matter has high signal intensity on short TR images, even though it has $12 \%$ less water than gray matter (11). The magnetization transfer technique enables semi

DOI: 10.9790/0853-1509080108 $\quad$ www.iosrjournals.org $\quad 1 \mid$ Page


quantitative characterization of tissue and pathologic entities, which could substantially improve the specificity of MR imaging $(11,12,13)$. By measuring the signal intensity with and without application of the pulses, magnetization transfer ratio (MTR) can be calculated. MTRs can be used to detect changes in the structural status of brain parenchyma that may or may not be visible with standard MR technique.

Infections of the central nervous system (CNS) and single CT enhancing lesions (SCTEL) are the major provoking factors in patients with acute onset simple or complex partial seizure(s) with or without secondary generalization.(1,9).Tuberculoma are usually more than $20 \mathrm{~mm}$, are associated with midline shift, raised intracranial tension, and progression. $(1,3,38)$. Basal meningitis with parenchymal lesions is highly suggestive of tuberculous etiology. Caseating tuberculoma with solid centre are hypo intense with hyper intense rim in T2 Weighted Images (38). Neurocysticercus granuloma are usually less than $20 \mathrm{~mm}$, no mass effect, no midline shift, no raised intracranial tension, and no progression. Cyst with dot inside (scolex) is diagnostic of Neurocysticercus granuloma. In Colloid vesicular stage, the cyst is hyper intense in T2WI. Degenerating cyst is hypo intense in T2WI when it mimics tuberculoma. Advanced MRI techniques like Magnetization Transfer Imaging, Apparent Diffusion Coefficient and Magnetic Resonance Spectroscopy are used to differentiate the two lesions.

Magnetization transfer ratio (22) is a quantitative measure of the MT effect on tissues. It is the degree of signal suppression of a given tissue compared with the conventional PD or T1-W image. The MT ratio (MTR) may be simply obtained by collecting a pair of identical images (PD or T1-W), one with and one without MT saturation.

\section{For each region of interest (ROI), MTR is calculated from the two images using the formula:} $\mathrm{MTR}=(\mathrm{Mo}-\mathrm{Ms}) \times 100$

Mo

Where Mo, Ms represent the signal intensity with the saturation pulse off and on, respectively. The T2 hypo intense Tuberculoma had the lowest MT ratios relative to T2 isointense / hyperintense Tuberculoma. Pathologically, the hyper intense and isointense (invisible) lesions are the solid granulomas with minimal or no caseation (5). With increased caseation, lesions tend to appear hypo intense on T2-weighted images. The caseation in a tuberculoma is composed predominantly of lipids and amino acids, as has been documented with proton MR spectroscopy (25). Lipids are not known to show MT (26). The presence of lipids as the major macromolecular constituent of the caseating granulomas as compared with noncaseating granulomas is probably responsible for the lower MT ratios in the former. T2 hypo intense tuberculomas have shown much lower MT ratios as compared with gray and white matter, and are prominent on precontrast MT-SE images. T2 hypo intense cysticercus granulomas have a significantly higher MT ratio than do similar-appearing tuberculomas, and are of low visibility, especially when located in the white matter (8). Quantitative MT ratios may help differentiate these similar-appearing infective granulomas and thus aid in the lesion-specific targeting of appropriate therapy. (8)

\section{Materials and Methods}

The study was started after the approval from the Hospital Ethical Committee. The study group included 43 consecutive patients with intra cranial ring enhancing lesions, were referred to the Barnard Institute of Radiology, Madras Medical College, Chennai for MRI Brain in June 2010 to November 2010. Patients clinically suspected of having intracranial granulomas, who had ring enhancing lesions in Contrast Enhanced Computed Tomography (CECT) or Magnetization Resonance Imaging of Brain were included in the study. All patients with calcified granuloma, patients with a known primary malignancy, patients with claustrophobia and patients with other contraindications for MRI (pacemaker implants, cochlear implants etc) were excluded from the study.

\section{Mr image acquisition:}

All patients provided the informed consent for performing additional sequences during imaging. Brief history regarding the nature and duration of illness, treatment history, significant past and personal history is taken from all patients. The study was performed in 1.5 Tesla SIEMENS MAGNETOM SYMPHONY MRI scanner. Conventional spin echo, non contrast T1-Weighted (TR 1000, TE 14) axial MR images with and without off - resonance saturation pulse and T2WI were taken. 3D CISS sequence was taken in axial plane at the site of the lesion. All images were obtained in axial plane with $5 \mathrm{~mm}$ slice thickness and a base resolution of 192 x 256 matrixes.

\section{Data Analysis}


Images were analyzed by two separate radiologists. Signal intensity from the rim of the granuloma was obtained with a single pixel from the conventional T1WI (without an off resonance pulse) and MT images (with an off resonance pulse). For each region of interest (ROI), MTR is calculated from the two images using the formula. Consistency and reliability of the measurements were confirmed by obtaining the values repeatedly. The final diagnosis of tuberculosis was based on typical CSF features (cellularity and biochemistry), histopathological examination and response to specific treatment.

The diagnosis of Neurocysticercosis was based on the presence of scolex in a cyst in ps $3 \mathrm{~d} / 3 \mathrm{~d}$ CISS sequence in MR Images. As per the Revised diagnostic criteria for Neurocysticercosis, the presence of multiple cystic lesions with or without scolex on CT or MRI is an Absolute criterion for the definitive diagnosis of neurocysticercosis. Presence of additional findings like infarct, meningeal thickening, basal meningitis were also noted. The MT Ratio from the cortical and deep gray and white matter from frontal, parietal, occipital and temporal regions, head of caudate nucleus and thalamus was also obtained (Fig 1).

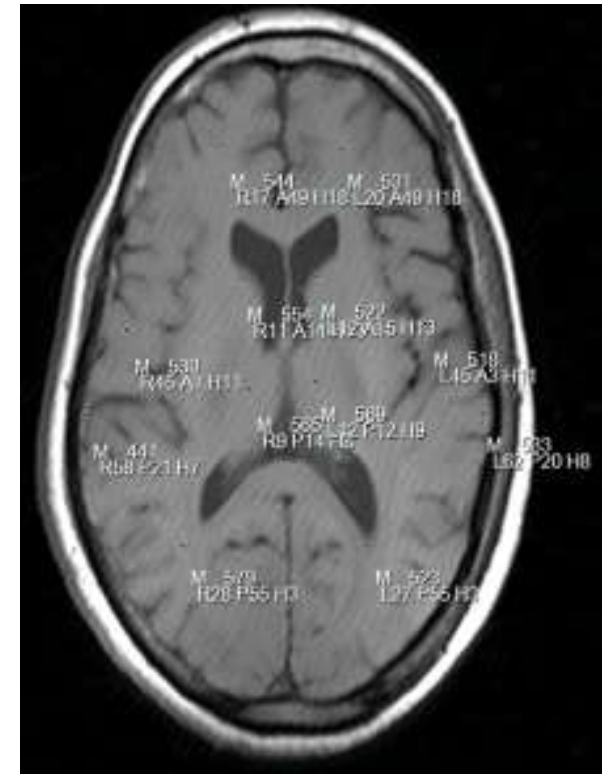

Fig 1a

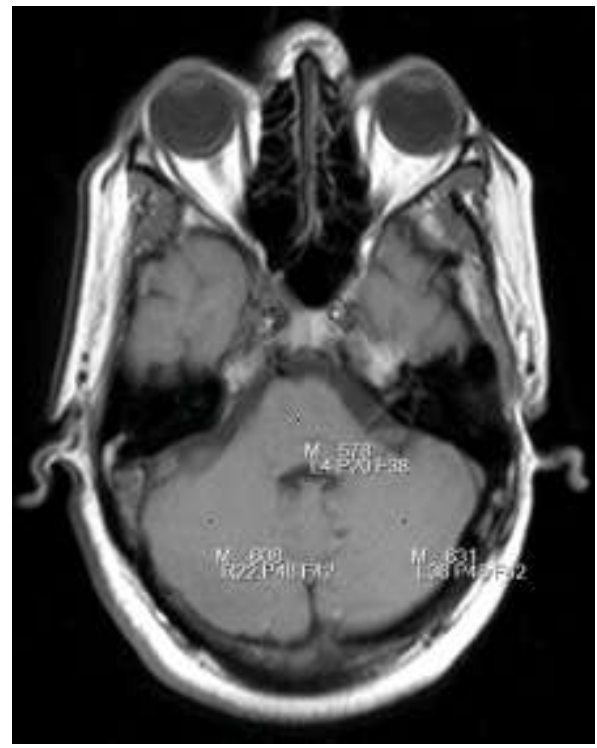

Fig 1c

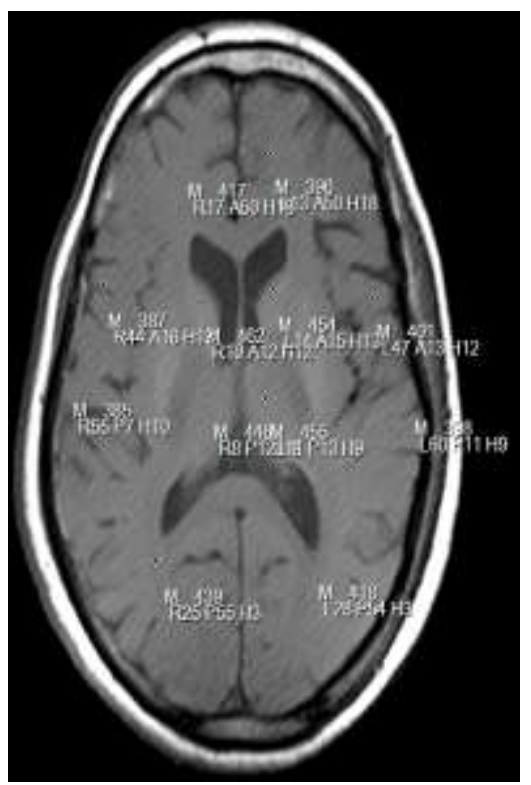

Fig 1b

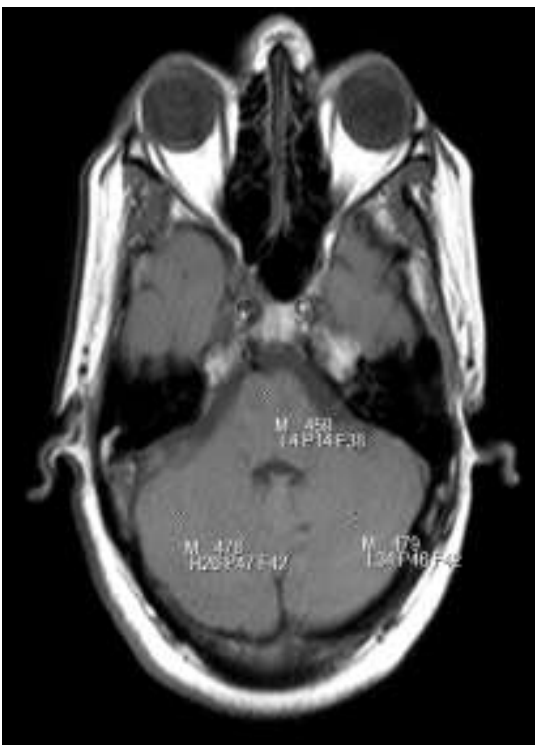

Fig 1d

Fig 1 a, b, c, d- MT Ratio from the cortical and deep gray and white matter from frontal, parietal, occipital and temporal regions, head of caudate nucleus and thalamus.

The lesions were grouped as Tuberculoma (Fig 2), degenerative NCC (Fig 3). (T2 hypointense / hyperintense) and cystic NCC (T2 hyper intense and follows CSF intensity). 


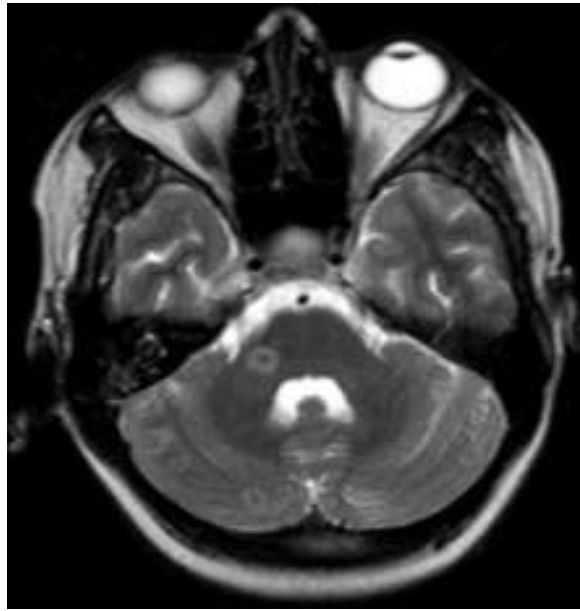

Fig 2a

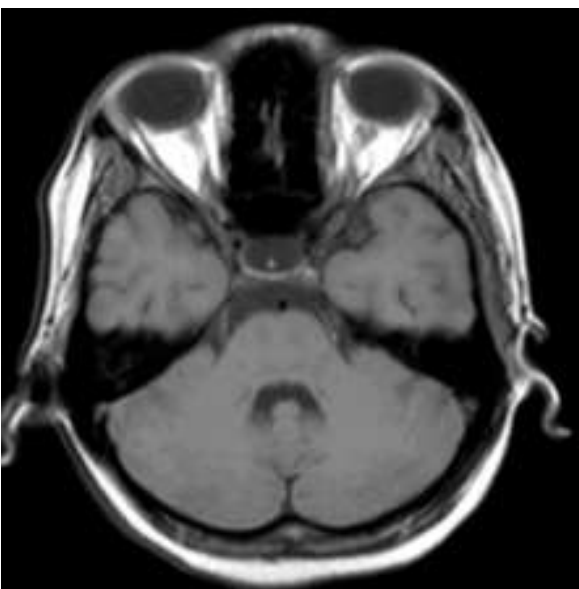

Fig 2c

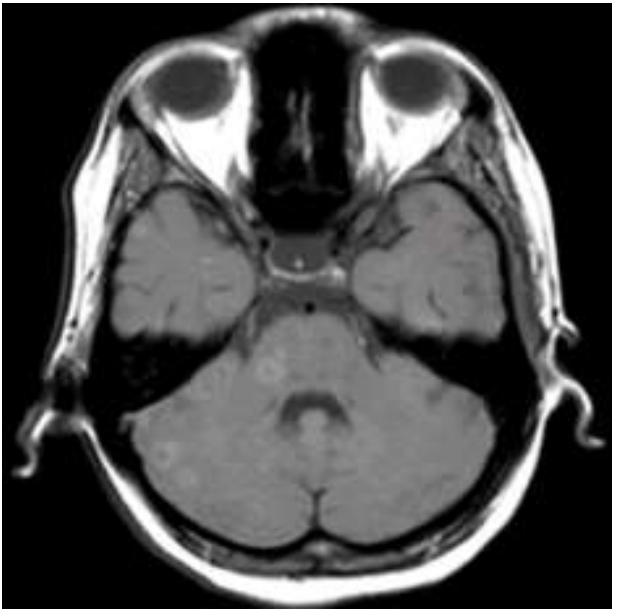

Fig 2b

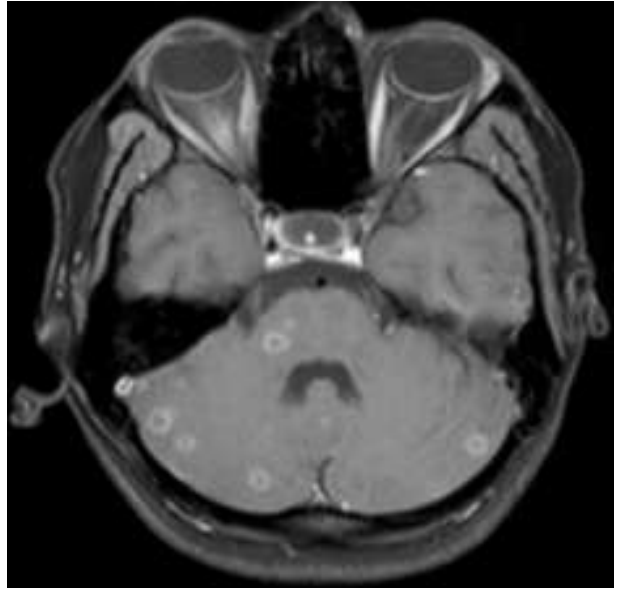

Fig 2d

Fig 2a- T2w MRI Showing Multiple T2 hypointense tuberculoma which have hypointense core with surrounding hyperintense rim

Fig 2c T1 W MT SE image shows the granuloma more conspicuous when compared to T1WI. Fig 2d - T1W Pre and Post contrast showing ring enhancing lesions.

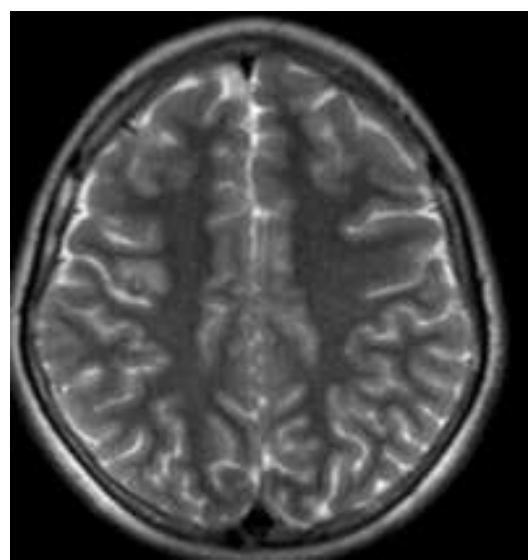

Fig 3a

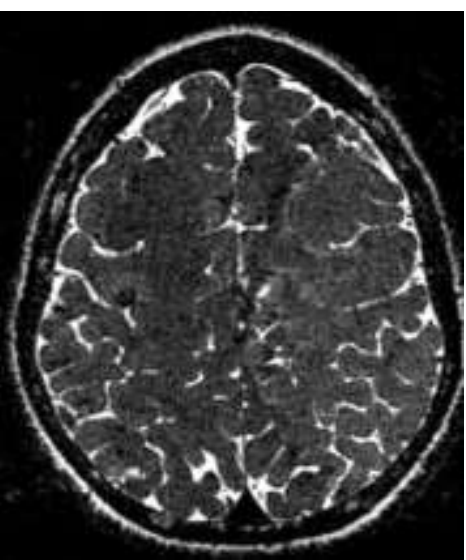

Fig 3b 


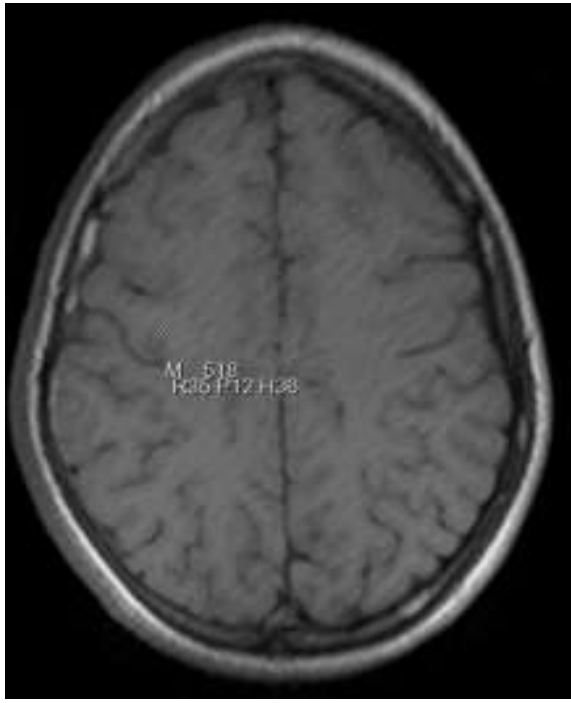

Fig 3c

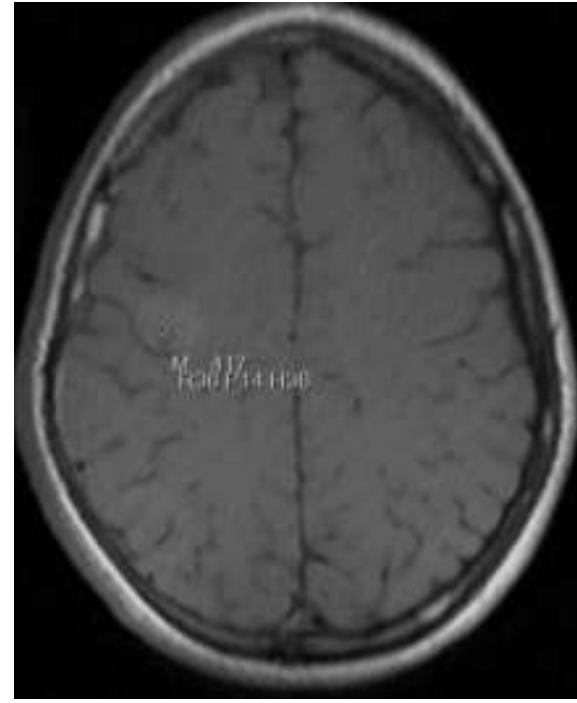

Fig 3d

Fig 3a,b, c,d -Axial T2W, 3D CISS, T1W, T1/MT SE W images at the level of centrum semiovale showing degenerative Neurocysticercosis.

The MT Ratio obtained from the rim of the lesion was tabulated and analyzed using ANOVA method. The Mean MT ratios were obtained for each of the three category with Standard Deviation from the Mean.

\section{Results}

A total of 76 granulomas from 43 patients with an age range from 6 to 72 years were analyzed of which 26 patients were men and 17 patients were women. Of these 76 granulomas, 41 were Tuberculomas analyzed in 21 patients and 35 were Neurocysticercous lesions analyzed in 22 patients. Cystic vesicular Neurocysticercus were 21 and degenerating Neurocysticercus were 14. 31 patients had single ring enhancing lesion. Multiple ring enhancing lesions were seen in 12 patients. Histopathological evidence of tuberculosis was present in one patient with Tuberculous abscess in the left middle cerebellar peduncle. The typical radiological features of miliary pulmonary tuberculosis were present in 4 patients. One patient had caries spine. Rest of the patients had positive chest x-ray for pulmonary tuberculosis. 13 patients had solitary lesions in brain and had typical features of tuberculoma in conventional MRI. Follow up after 2 months of treatment showed clinical improvement and reduction in the size of the lesion or the surrounding vasogenic edema.

The MT Ratio of the normal white matter and deep gray matter from healthy controls measured $23.72 \pm 2.36$ and 17.58 \pm 3.52 respectively. The data was taken for comparison with tuberculomas and T2 hypointense cysticercous granulomas. Mean MTR for tuberculoma is 16.6 +/- 3.1(15.6-17.56).

Descriptives

\begin{tabular}{|c|c|c|c|c|c|c|c|c|}
\hline & \multirow[b]{2}{*}{$\mathrm{N}$} & \multirow[b]{2}{*}{ Mean } & \multirow[b]{2}{*}{ Std. Deviation } & \multirow[b]{2}{*}{ Std. Error } & \multicolumn{2}{|c|}{$\begin{array}{l}\text { 95\% Confidence Interval for } \\
\text { Mean }\end{array}$} & \multirow[b]{2}{*}{ Mnimum } & \multirow[b]{2}{*}{ Maximum } \\
\hline & & & & & Low er Bound & Upper Bound & & \\
\hline NCC - VESCICULAR & 21 & 10.9000 & 2.82981 & .61752 & 9.6119 & 12.1881 & 2.90 & 14.70 \\
\hline NCC - DEGENERA TING & 14 & 20.8500 & 3.57378 & .95513 & 18.7866 & 22.9134 & 16.70 & 28.00 \\
\hline TUBERCULOMA & 41 & 16.5944 & 3.08239 & .48139 & 15.6215 & 17.5673 & 6.00 & 22.10 \\
\hline Total & 76 & 15.8049 & 4.60920 & .52871 & 14.7516 & 16.8581 & 2.90 & 28.00 \\
\hline
\end{tabular}

Table 1- Mean MTR of ring enhancing lesions

\begin{tabular}{|c|c|c|c|c|c|}
\hline & $\begin{array}{l}\text { Sum of } \\
\text { Squares }\end{array}$ & df & Mean Square & $\mathrm{F}$ & Sig. \\
\hline Betw een Groups & 887.116 & 2 & 443.558 & 45.848 & .000 \\
\hline Within Groups & 706.236 & 73 & 9.674 & & \\
\hline Total & 1593.353 & 75 & & & \\
\hline
\end{tabular}

Table 2- ANOVA tests Showing difference in MTR values between Tuberculous lesions \& NCC is statistically significant $\mathrm{P}$ value $<.05$ 
There were 21 innocuous cystic lesions (representing vesicular stage of NCC) which showed mean MTR of 10.9 \pm 2.8 (9.6 - 12.18). 14 lesions with imaging features suggestive of degenerative cysts showed MTR of $20.8 \pm$ 3.5 (18.7 - 22.9). There were 3 lesions which were hypointense on T2WI which showed a mean MTR which was significantly higher $(23.725 \pm 3)$.

\begin{tabular}{|c|c|c|c|c|c|c|}
\hline \multirow[b]{2}{*}{ (1) DIAGNOSIS } & \multirow[b]{2}{*}{ (J) DIAGNOSIS } & \multirow{2}{*}{$\begin{array}{c}\text { Mean } \\
\text { Difference } \\
(1-J)\end{array}$} & \multirow[b]{2}{*}{ Std. Error } & \multirow[b]{2}{*}{ Sig. } & \multicolumn{2}{|c|}{$95 \%$ Confidence Interval } \\
\hline & & & & & Low er Bound & Upper Bound \\
\hline \multirow[t]{2}{*}{ NCC - VESCICULAR } & NCC - DEGENERA TING & $-9.95000^{*}$ & 1.07318 & .000 & -12.5175 & -7.3825 \\
\hline & TUBERCULOMA & $-5.69439^{*}$ & .83466 & .000 & -7.6913 & -3.6975 \\
\hline \multirow[t]{2}{*}{ NCC - DEGENERA TING } & NCC - VESCICULAR & $9.95000^{*}$ & 1.07318 & .000 & 7.3825 & 12.5175 \\
\hline & TUBERCULOMA & $4.25561^{*}$ & .96281 & .000 & 1.9522 & 6.5591 \\
\hline \multirow[t]{2}{*}{ TUBERCULOMA } & NCC - VESCICULAR & $5.69439^{*}$ & .83466 & .000 & 3.6975 & 7.6913 \\
\hline & NCC - DEGENERA TING & $-4.25561^{*}$ & .96281 & .000 & -6.5591 & -1.9522 \\
\hline
\end{tabular}

Table 3 - Post Hoc Tests, When multiple comparisons are made, the mean difference between the ratio of the three groups is significant.

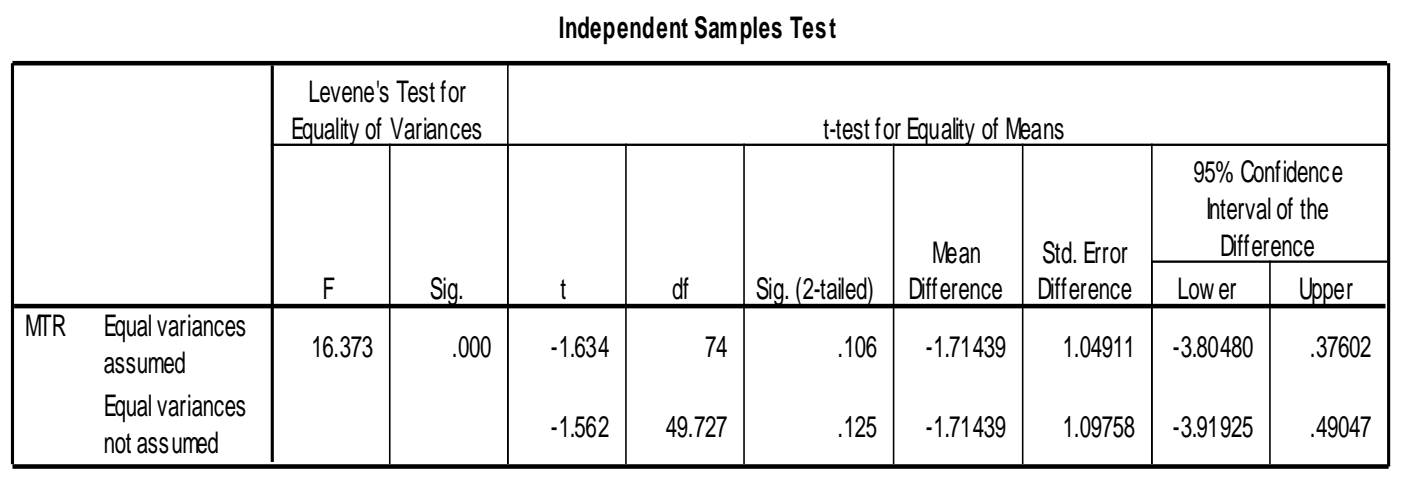

Table 4 -T-Test, showing the group allocation is correct.

\section{Discussion}

MTR was calculated from the rim of the lesion. In the study of Rakesh Gupta et al (8), it is stated there was no significant difference between the MT ratios in the core and the rim of Tuberculous lesions. Mean MTR of tuberculoma in our study was $16.6 \pm 3.7$. Using MT Ratio of $16.6 \pm 3.7(12.9-20.3)$, the sensitivity for detecting tuberculoma was $78 \%$, specificity was $73 \%$ and positive predictive value was $78 \%$. In addition, Tuberculomas were better seen in precontrast MT SE images compared to T1WI and T2WI especially T2 hypointense Tuberculomas. More lesions were detected on precontrast MT-SE images, since few lesions did not enhance after contrast injection. Contrast enhancement due to disruption of the blood-brain barrier depends on the activity of the lesion and inactive lesions may not enhance after contrast administration (8). Two study patients also had basal meningitis. In precontrast MTSE images, parenchymal hyper intensity along the surface of brain stem was visible. On contrast, there was enhancement of the basal meninges and cisternal tuberculoma.

35 Neurocysticercus lesions were analyzed in 22 patients. The size of the lesion ranged from $8 \mathrm{~mm}$ to $15.5 \mathrm{~mm}$. Intra ventricular cysts were larger in size. Demonstration of scolex within a cyst in CT/MRI is considered the absolute criteria for the diagnosis of Neurocysticercosis as per the revised diagnostic criteria of NCC. 3D CISS images demonstrate the cyst wall and scolex due to inherent high contrast resolution of the sequence. Degenerating NCC cysts show hypo intense center with hyper intense rim in MT images. There were 21 cystic lesions (representing vesicular stage of NC) which showed MTR of $10.9 \pm 2.8(9.6-12.18)$. There were 3 granuloma which were hypointense on T2WI which showed MTR which was significantly higher (23.725 \pm 3$) .14$ lesions with imaging features suggestive of degenerative cysts showed MTR of $20.8 \pm 3.5$ (18.7 $-22.9)$

The difference in the MTR values of tuberculous \& NCC lesions is illustrated by box chart in figure 4 


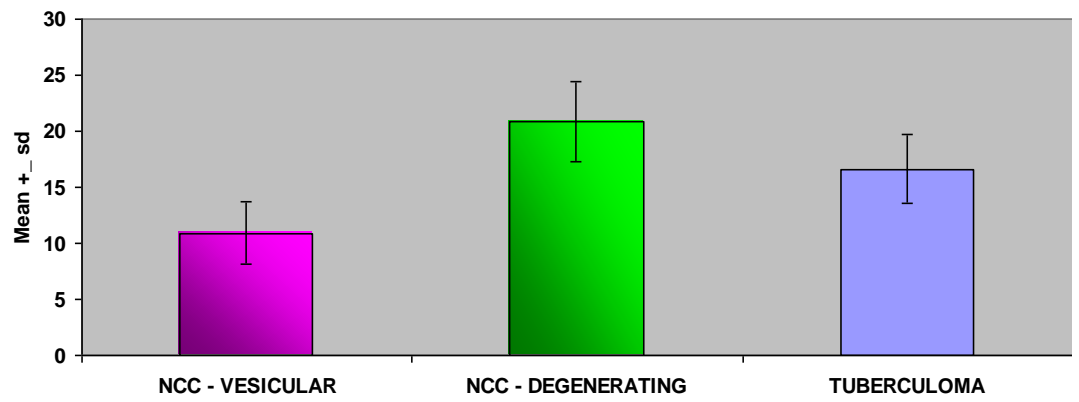

Fig 4- Efficacy of MTR in differentiating tuberculous lesions versus NCC

According to the study of Rakesh Gupta et al, the tuberculous lesions with a hypointense core on T2weighted images had MT ratios of $23.8 \pm 1.76$ in the rim and $24.2 \pm 3.1$ in the core. In our study, the mean MTR was $16.6 \pm 3.7$ for Tuberculoma. There are several key parameters in the off-resonance saturation transfer technique that can alter the quantitative and qualitative MTC. The principle determinants being the base sequence used, characteristics of the saturating pulse (pulse type, power, duration, time between pulses, duty cycle i.e. number of pulses per TR, bandwidth, its effective flip angle), offset frequency of the saturating pulse, and field strength. All of these characteristics alter the degree of saturation and Magnetization transfer ratio obtained and must be known before quantitative comparisons can be made between clinical studies. (22). So, machine parameters could be the reason for the variability of MTR measured in both studies.

In 1998, Kathuria MK et al (28) measured the magnetization transfer ratio (MTR) in different stages of neurocysticercosis. The visibility of a lesion on MT-SE sequence was dependent on its MTR and its location at a particular site (cortical gray matter, white matter, or deep gray matter). The difference in MTR of the lesion and the surrounding brain parenchyma decides the resulting contrast and visibility of the lesion Innocuous cystic lesions, which were hyperintense on T2-weighted images, did not show any MT (MTR = 5.10+/-1.2), whereas degenerating T2 hyperintense lesions showed MTR of 26.40 \pm 2.7 . Nondegenerating and degenerating scolices showed an intermediate MTR of $21.7 \pm 3.3$ and $15.0 \pm 4.5$, respectively.

\section{Limitations of the study}

The total number of the lesions analysed include 41 tuberculoma and 35 Neurocysticercus. A total of 107 tuberculomas in seven patients were analyzed in the study by Rakesh Gupta et al (8). The no of lesions enrolled in this study were less than the previous study conducted. This could account for the wide confidence interval noticed in this study. Magnetization transfer ratio obtained for white and grey matter as well as tuberculoma and NCC in our study were less than the previous studies. This is probably due to technical and machine parameters. Hence MTR has to be standardized for each machine and MTR between two different studies cannot be compared. Histopathalogical results are available for only one lesion.

\section{Conclusion}

MT ratio can be used to differentiate T2 hypointense NCC and Tuberculoma. MT Ratio of Tuberculoma is $16.6 \pm 3.1$ and MT Ratio of T2 hypointense NCC is 23.725. T2 invisible Tuberculomas are better visualized in MT images. Hence disease load can be better assessed using MT images. Presence of associated meningeal disease can be demonstrated using precontrast MT SE Images in Tuberculoma. Including precontrast MT SE T1 Weighted sequence in our routine MRI Brain protocol for granuloma imaging can help us differentiate T2 hypointense NCC and Tuberculoma, better visualize T2 invisible Tuberculomas and detect presence of associated meningeal disease.

\section{References}

[1]. Ravindra Kumar Garg et al, Single Enhancing Computerized Tomography-Detected Lesion in Immunocompetent Patient,Journal of Neurosurgery June 2002 Volume 12, Number 6.

[2]. AP Jain, Rajnish Joshi, Tejal Lathia, Single Small Enhancing Computed Tomography Lesion: A Review, JIACM 2005; 6(2): 11421.

[3]. Rajshekhar V, Haran RP, Prakash GS, et al, Differentiating solitary small cysticercus granulomas and tuberculomas in patients with epilepsy. Clinical and computerized tomographic criteria, J Nerurosurg 78:402-407, 1993.

[4]. Chopra JS, Sawhney IMS, Suresh N, et al: Vanishing CT lesions in epilepsy. J Neurol Sci 107:40-49, 1992

[5]. Garg RK, Nag D: Single enhancing CT lesions in Indian patients with seizures: clinical and radiological evaluation and follow-up. J Trop Pediatr 44:204-210, 1998

[6]. Garg RK, Singh MK, Misra S: Single-enhancing CT lesions in Indian patients with seizures: a review. Epilepsy Res 38: 91-104, 2000

[7]. J.M.K Murthya, Ravi Yangalab, Acute symptomatic seizures - incidence and etiological spectrum: a hospital-based study from South India, Volume 8, Issue 3, Pages 162-165 (May 1999). 
[8]. Rakesh K. Gupta, Manoj K. Kathuria, and Sunil Pradhan, Magnetization Transfer MR Imaging in CNS Tuberculosis; AJNR Am J Neuroradiol 20:867-875, May 1999.

[9]. Chandy MJ, Rajshekhar V, Prakash S, et al: Cysticercosis causing single, small CT lesions in Indian patients with seizures. Lancet 1:390-391, 1989

[10]. Rakesh K. Gupta, N. Husain, M.K. Kathuria, S. Datta, R.K.S. Rathore, M. Husain, Magnetization Transfer MR Imaging Correlation with Histopathology in Intracranial Tuberculomas; Clinical Radiology, Volume 56, and Issue 8, Pages 656-663.

[11]. Robert I. Grossman, MD, John M. Gomori, MD, Karen N. Ramer, BA, FrankJ. Lexa, MD, Mitchell D. Schnall, MD, PhD,Magnetization Transfer: Theory and Clinical Applications in Neuroradiology; RadioGraphics 1994; 14:279-290

[12]. Pulsed Magnetization Transfer Imaging : Evaluation Of Technique Simon J Graham, PhD, R Mark Henkeiman, PhD; Radiology 1999; $202903-910$

[13]. Jinkins JR, Gupta R, Chang KH, Carbajal JR. MR imaging of central nervous system tuberculosis. Radiol Clin North Am 1995;33:771-786

[14]. Mehta RC, Bruce Pike G, Enzmann DR. Magnetization transfer MR of the normal adult brain. AJNR Am J Neuroradiol 1995; 16:2085-2091

[15]. Edelman RR, Ahn SS, Chien D, et al. Improved time-of-flight MR angiography of the brain with magnetization transfer contrast. Radiology 1992;184:395-399

[16]. Wolff S, Balaban R. Magnetization transfer contrast (MTC) in magnetic resonance imaging. Magn Reson Med 1989;10:135-144

[17]. Balaban RS, Ceckler TL. Magnetization transfer contrast in magnetic resonance imaging. Magn Reson Q 1992;8:116-137

[18]. Yeung HN AA. Magnetization transfer contrast with periodic pulsed saturation; Radiology 1992;183:209-2147.

[19]. Muller RN, Marsh MJ, Bemardo MI, Lauterbur PC. True 3-D imaging oflimbs by NMR zeugmatography with off-resonance irradiation. EurJ Radiol 1983; 3:286-290.

[20]. Wolff SD, Balaban RS. Magnetization transfer contrast (MTC) and tissue water proton relaxation in vivo. Magn Reson Med 1989; 10:135-144.

[21]. Wolff SD, EngJ, Balaban RS. Magnetization transfer contrast: method for improving contrast in gradient-recalled-echo images. Radiology 1991;179:133-137.

[22]. R Gupta,2002, Magnetization transfer MR imaging in central nervous system infections, Indian Journal of Radiology and Imaging Volume : 12 , Issue : 1, Page : 51-58

[23]. Agarwal A, Raghav S, Husain M, Kumar R, Gupta RK. Epilepsy with focal cerebral calcification: Role of magnetization transfer MR imaging. Neurol India 2004;52:197-9.

[24]. Central Nervous System Tumor, Infection, and Infarction: Detection with Gadolinium-enhanced Magnetization Transfer MR Imaging' Radiology 1995; 195:41-46.

[25]. Gupta RK, Roy R, Poptani H, et al. Finger printing of Mycobacterium tuberculosis in intracranial tuberculomas using in vivo, ex vivo and in vitro proton MR spectroscopy. Magn Reson Med 1996;36:829-833.

[26]. Woff SD, Balaban RS. Magnetization transfer contrast (MTC) and tissue water proton relaxation in vivo. Magn Reson Med 1989;10:135-144.

[27]. Rakesh K Guptaa, Mazhar Husainb, Devendra K Vatsalb, Rajesh Kumara, Sanjeev Chawlaa, Nuzhat Husain, Comparative evaluation of magnetization transfer MR imaging and in-vivo proton MR spectroscopy in brain tuberculomas; Magnetic resonance imaging, volume 20, Issue 5, Pages 375-381 (June 2002).

[28]. Kathuria MK, Gupta RK, Roy R, Gaur V, Husain N, Pradhan S.Measurement of magnetization transfer in different stages of neurocysticercosis; J Magn Reson Imaging 1998;8:473-479.

[29]. R.Gupta, Comparative evaluation of magnetization transfer MR imaging and in-vivo proton MR spectroscopy in brain tuberculomas, Magnetic Resonance Imaging, Volume 20, Issue 5, Pages 375-381.

[30]. M. K. Vasudev, P. N. Jayakumar, S. G. Srikanth, K. Nagarajan and A. MohantyQuantitative Magnetic Resonance Techniques in the Evaluation of Intracranial Tuberculomas: Acta radiologica 2007, Vol. 48, No. 2, Pages 200-206

[31]. Intracranial tuberculomas: MRI signal intensity correlation with histopathology and localised proton spectroscopy. Gupta RK, Pandey R, Khan EM, Mittal P, Gujral RB, Chhabra DK. Magn Reson Imaging. 1993;11(3):443-9.

[32]. Khanna PC, Godinho S, Patkar DP, Pungavkar SA, Lawande MA. MR spectroscopy-aided differentiation:"Giant" extra-axial tuberculoma masquerading as meningioma; AJNR Am J Neuroradiol 2006;27:1438-40.

[33]. Shindo A, Honda C, Baba Y. A case of an intracranial tuberculoma, mimicking meningioma that developed during treatment with anti-tuberculous agents. No Shinkei Geka 1999;27:837-41

[34]. Bauer J, Johnson RF, Levy JM, Pojman DV, Ruge JR. Tuberculoma presenting as an en plaque meningioma. Case report. J Neurosurg 1996;85:685-8.

[35]. 33. A Kuruvilla, J D Pandian, M Nair, V V Radhakrishnan, S Joseph, Neurocysticercosis: A Clinical and Radiological Appraisal from Kerala State, South India; Singapore Med J 2001 Vol 42(7) : 297-303.

[36]. Professor Arturo Carpio a et al, The Lancet Infectious Diseases, Neurocysticercosis: an update; Volume 2, Issue 12, Pages 751 762, December 2002.

[37]. HR Martinez et al., MR imaging in neurocysticercosis: a study of 56 cases ; American Journal of Neuroradiology, Vol 10, Issue 5 1011-1019.

[38]. Srikanth Subbamma Govindappa et al,Improved Detection of Intraventricular Cysticercal Cysts with the Use of Three-dimensional Constructive Interference in Steady State MR Sequences; AJNR Am J Neuroradiol 21:679-684, April 2000.

[39]. R.Gupta, M.Prakash, A.Mishra, M.Husain, K.Prasad, N.Husain, Role of diffusion weighted imaging in differentiation of intracranial tuberculoma and tuberculous abscess from cysticercus granulomas-a report of more than 100 lesions European Journal of Radiology, Volume 55, Issue 3, Pages 384-392.

[40]. Rajshekhar V, Chandy MJ. Validation of diagnostic criteria for solitary cysticercus granuloma in patients presenting with seizures. Acta Neurol Scand 1997;96:76-81. 